\title{
Age-related Changes in Local Water and Protein Content of Human Eye Lenses Measured by Raman Microspectroscopy
}

\author{
ITTE SIEBINGA $A^{a, b}$, GIJS F. J. M. VRENSEN ${ }^{* *}$, FRITS F. M. DE MUL ${ }^{b}$, AND JAN GREVE \\ a Netherlands Ophthalmic Research Institute, P.O. Box 12141, 1100 AC Amsterdam, The Netherlands, \\ and ${ }^{b}$ Department of Applied Physics, Twente University of Technology, P.O. Box 217. \\ 7500 AE Enschede, The Netherlands
}

(Received 11 August 1989 and accepted in revised form 23 January 1990)

\begin{abstract}
The Raman microspectroscopic method was used to determine the local water and protein content in human lenses. In 18 lenses of varying age position-defined water/protein content measurements were carried out along the visual and the equatorial axis.

A main characteristic of the human lens is its constant and relatively low protein content. In addition this constant nuclear value is reached within a short distance from the capsular surface. For statistical analysis of age-related changes the data points in individual lenses were piecewise linearized. (1) The mean nuclear water content was calculated from the data points in the inner $80 \%$ of the visual axis. (2) The steep drop in water content was linearized using a least-squares linear regression approach. The distance between lenticular surface and the intersection of the regression line with the line representing the nuclear mean was denominated as surface layer width.

It proved that: (i) the mean nuclear water content significantly increased with age, (ii) the width of the surface layer was age independent in the anterior and posterior poles of the visual axis, and (iii) in the equatorial axis the surface layer width significantly decreased with age.

Seven human lenses with small opaque spots were also investigated. The opaque spots proved to have a normal-for-site water content and some of them were flanked at their capsular side by a zone with a high-for-site water content.

The correlation between protein content and refractive index and the observed decrease in nuclear protein content in aging human lenses can be taken as strong evidence that upon aging the refractive index of a major part of the human lens is reduced. The implications of this decrease is discussed with the respect to the problem known as the lens Paradox, i.e. the discrepancy between the theoretically expected age-related loss of far vision due to changes in lens curvature and axial position in the eye and the actually observed loss in near vision upon age.

Key words: Raman spectroscopy; human lens; water content; protein content; aging; cataractous opacities.
\end{abstract}

\section{Introduction}

Many studies have been devoted to the relationship between water content and aging in human lenses. To investigate the total water content of eye lenses different methods have been used. Fisher and Pettet (1973) and Lahm. Lee and Bettelheim (1985) determined the total water content by vacuum dehydration and Deussen and Pau (1989) used a freezedrying technique to determine the total lens water. Also the lenticular water content in association with the occurrence of opacities has been investigated thoroughly (Bettelheim, et al., 1986a.b; Deussen and Pau, 1989). Despite the reliability of the methods used in these studies, the main disadvantage of these methods is the rough subdivision of the lens in, for example, cortical and nuclear parts. Studying the water gradient in human lenses and studying the water content in small opaque spots requires strictly local measurement and small measuring volumes. Local measurements of the water content in lenses have been carried out by Bours, Födisch and Hockwin

\footnotetext{
* For correspondence.
}

(1987), by using a microsectioning technique. Fagerholm. Philipson and Lindström (1981) determined the protein distribution in human lenses by a quantitative microradiographic method.

Another method with the ability to measure very small volumes is Raman microspectroscopy. In a previous study (Huizinga et al., 1989) Raman microspectroscopy proved to be a reliable method for the measurement of the absolute water content of small volumes on defined positions in the lens. In another study (Bot et al., 1989) the advantages of using lens slices were pointed out, which improves the accuracy of positioning and addiiionally enables Raman analysis of old human lenses with their prominent fluorescence.

The aim of the present study is to reinvestigate position-defined age-related changes in water content in human lenses, using the Raman microspectroscopic method. A second goal is to investigate the water content inside and around small opaque spots.

\section{Materials and Methods}

The Raman spectra were recorded, as described 


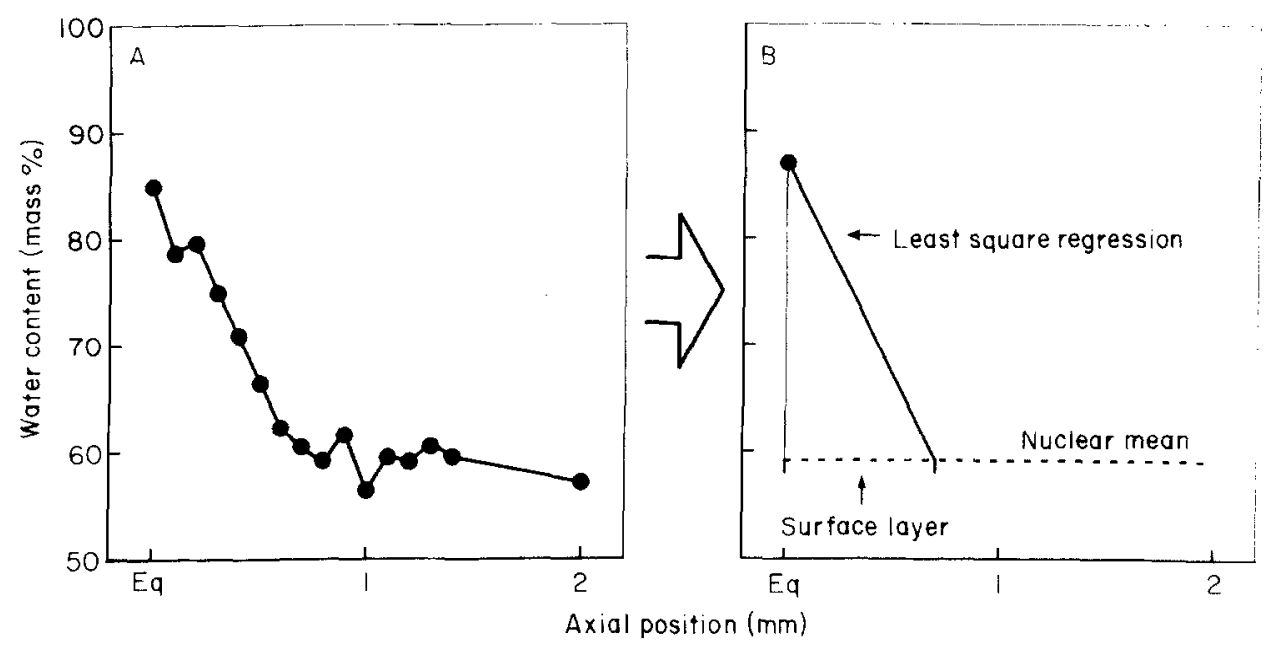

FIG. 1. Representation of the procedure used to linearize piecewise the water-content profiles. The water-content profile determined along the outermost $2 \mathrm{~mm}$ of the equatorial axis (A) is represented by straight lines (B). The horizontal dotted line represents the mean nuclear water content, calculated as the mean of all data points in the inner $80 \%$ of the visual axis. The uninterrupted straight line, representing the drop in water content, is calculated by least-squares regression from the data points at the most superficial position to about a point where the water content reached the nuclear value. Note the indication of the surface layer width. Eq : capsular side equator.
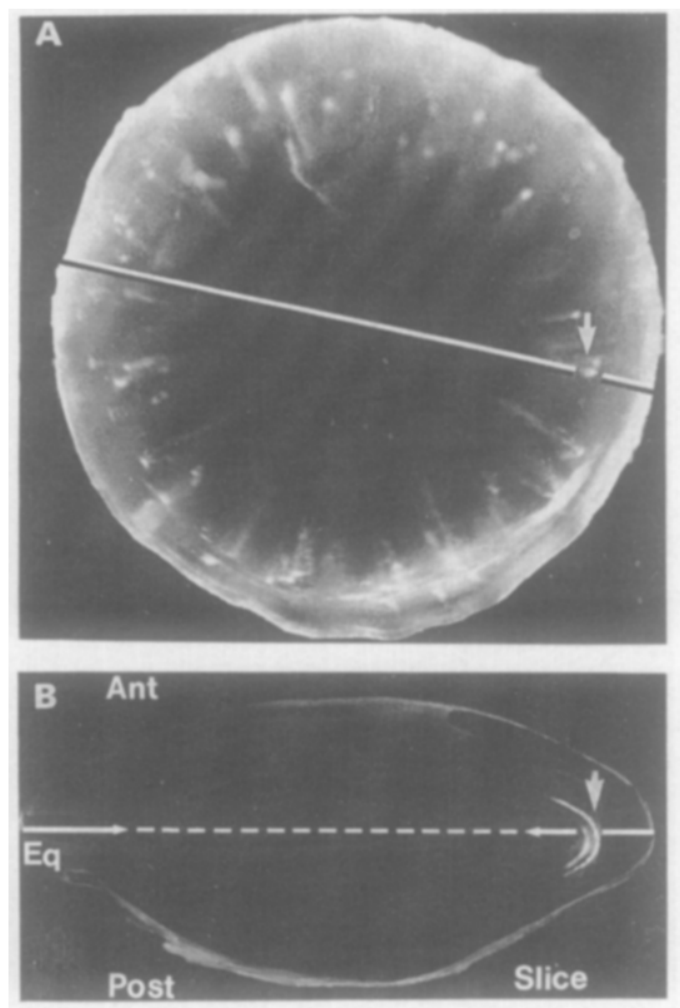

FIG. 2. Dark-field micrographs of an intact (A) and dissected (B) human lens with small opacities in the equatorial region. The straight line (A) indicates the plane of slice cutting passing through an opacity [ (arrows in (A) and (B)]. Measurements are carried out along the dotted line (B). Note the uninterrupted extension of the opacity in anterior and posterior direction.

earlier (Gijsbers et al., 1986) with a SPEX 1877B spectrometer, an EG\&G PAR OMA III multichannel detector, an Olympus BSM metal microscope, and a Coherent model 52 Argon-ion laser with Innova tube emitting at $514.5 \mathrm{~nm}$. The pixels of the multichannel detector were calibrated in $\mathrm{cm}^{-1}$ by measuring lines of an Argon calibration lamp, using a cubic fit procedure. The sensitivity variation over the multichannel detector range was eliminated by using a correction curve from a 'black-body' tungsten lamp. The effectively measured volume is defined by the dimensions of the entrance pupil of the spectrometer and the waist diameter of the laser beam. The effective pupil in the plane of the entrance slit is $100 \times 1500 \mu \mathrm{m}^{2}$. Waist diameter, focal depth and measured volume are $2 \mu \mathrm{m}$, $0.1 \mathrm{~mm}$ and $3 \times 10 \mu \mathrm{I}^{-3}$ respectively when using a $50 \times$ objective. The laser power at the sample is $25 \mathrm{~mW}$ and the resolution is $4 \mathrm{~cm}^{-1}$.

Eighteen clear human lenses were obtained from donor eyes sent for cornea transplantation. The lenses were extracted within 6-36 hr (mostly within $16 \mathrm{hr}$ ) post-Inortem. Imulediately after extraction the lenses were fixed in a solution of $0.08 \mathrm{M}$ cacodylate buffered $1 \%$ paraformaldehyde $(\mathrm{pH} 7 \cdot 3,310$ mosmol) during at least 1 week. Just before Raman analysis, lens slices of about $1 \mathrm{~mm}$ thickness were cut along the visual axis. The lens slices were put on a glass slide and covered with a coverglass of $0.17 \mathrm{~mm}$ thickness. A drop of a phosphate-buffered saline solution (PBS) was used to prevent drying. Raman spectra were obtained in the $2800-3700 \mathrm{~cm}^{-1}$ spectral region at different positions along the visual and equatorial axis. by using the horizontal stage controls. All spectra were recorded using exposure times of $100 \mathrm{sec}$ and were obtained $100 \mu \mathrm{m}$ below the surface of the lens slices. Along the outermost $0.5 \mathrm{~mm}$ of the visual axis. on the anterior and posterior side. Raman spectra were obtained at intervals of $50 \mu \mathrm{m}$. followed by intervals of $100 \mu \mathrm{m}$ up to $1 \mathrm{~mm}$ from the capsular side. The number of data points in the central part depended on the intensity of the fluorescence signal and varied from 1 to 6 . Since it was established that 


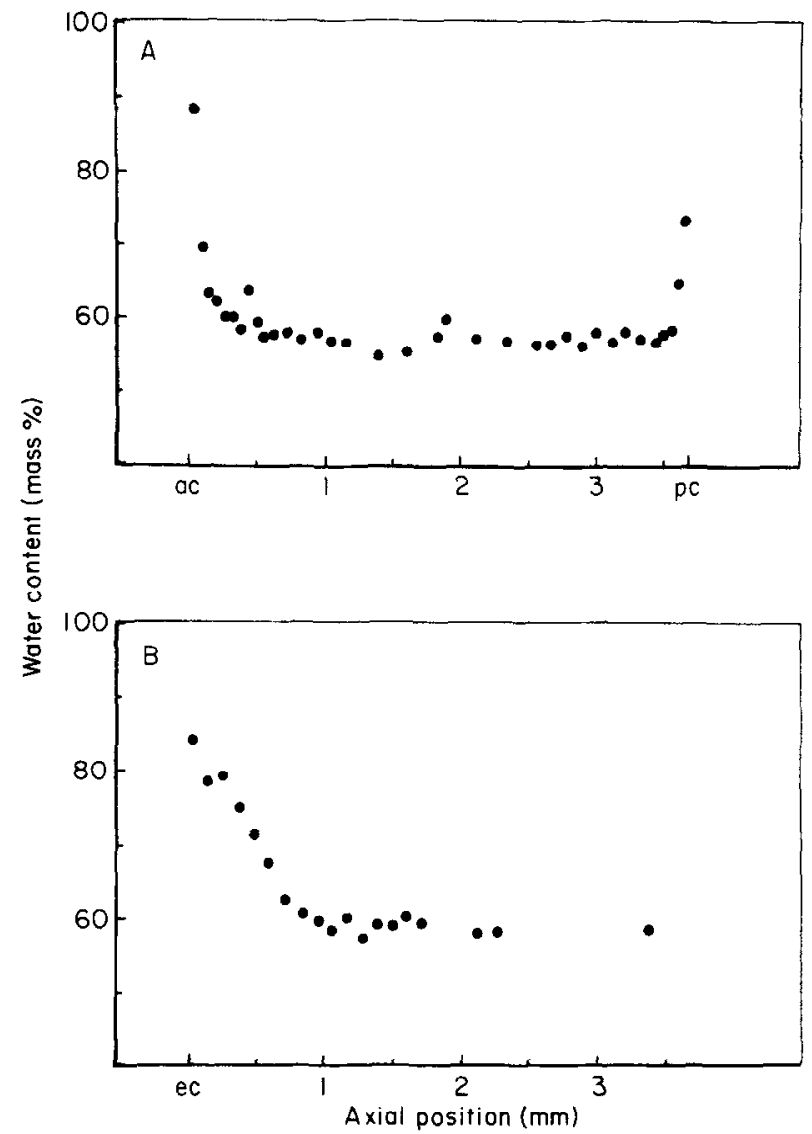

FIG. 3. Absolute water content along the visual $(A)$ and equatorial (B) axis of an eight-year-old human lens. ac: anterior capsular side; $\mathrm{pc}$ : posterior capsular side; ec: equatorial capsular side.

the nuclear water content was rather constant we restricted the measurements in the central part to 1 or 2 in cases of intensive fluorescence. Along the equatorial axis measurements were carried out unilaterally at intervals of $100 \mu \mathrm{m}$. From the Raman spectra the relative water-to-protein ratios, indicated by the intensity ratio I (3390)/I (2935), were calculated. These ratios were transformed to absolute water mass percentages, as described in a previous study (Huizinga et al., 1989). Since water and protein make up over $99 \%$ of the total mass of the lens, the protein content in percentage can simply be calculated as 100 minus percentage water content. For brevity we mainly indicate the water content in this paper.

In order to enable statistical analysis the data points were piecewise linearized. Since it turned out that in all lenses investigated the water content reached the nuclear value within $10 \%$ of the length of the visual axis, in both the anterior and the posterior pole, the mean value was calculated as the mean of all data points in the inner $80 \%$ of the visual axis. The data points representing the decrease in water content along the anterior/posterior axis and along the equatorial axis of individual lenses showed a considerable spread around a linear dependence. This may be due to very restricted variations or due to small inaccuracies in the measurement. Therefore we calculated by least-squares regression a straight line, representing the water content gradient, from the data points at the most superficial position to about a point where the water content reached the mean nuclear value. The mean nuclear value is represented by a horizontal line. We considered the value of the position of the intersection point within the lens obtained in this way, as a measure for the width of the surface layer where the water content is still decreasing to the value in the nucleus. A representation of this linearization procedure is shown in Fig. 1.

In addition. we studied seven human lenses with small opaque spots in the equatorial region. In Fig. 2(A) a dark-field micrograph is shown of an intact

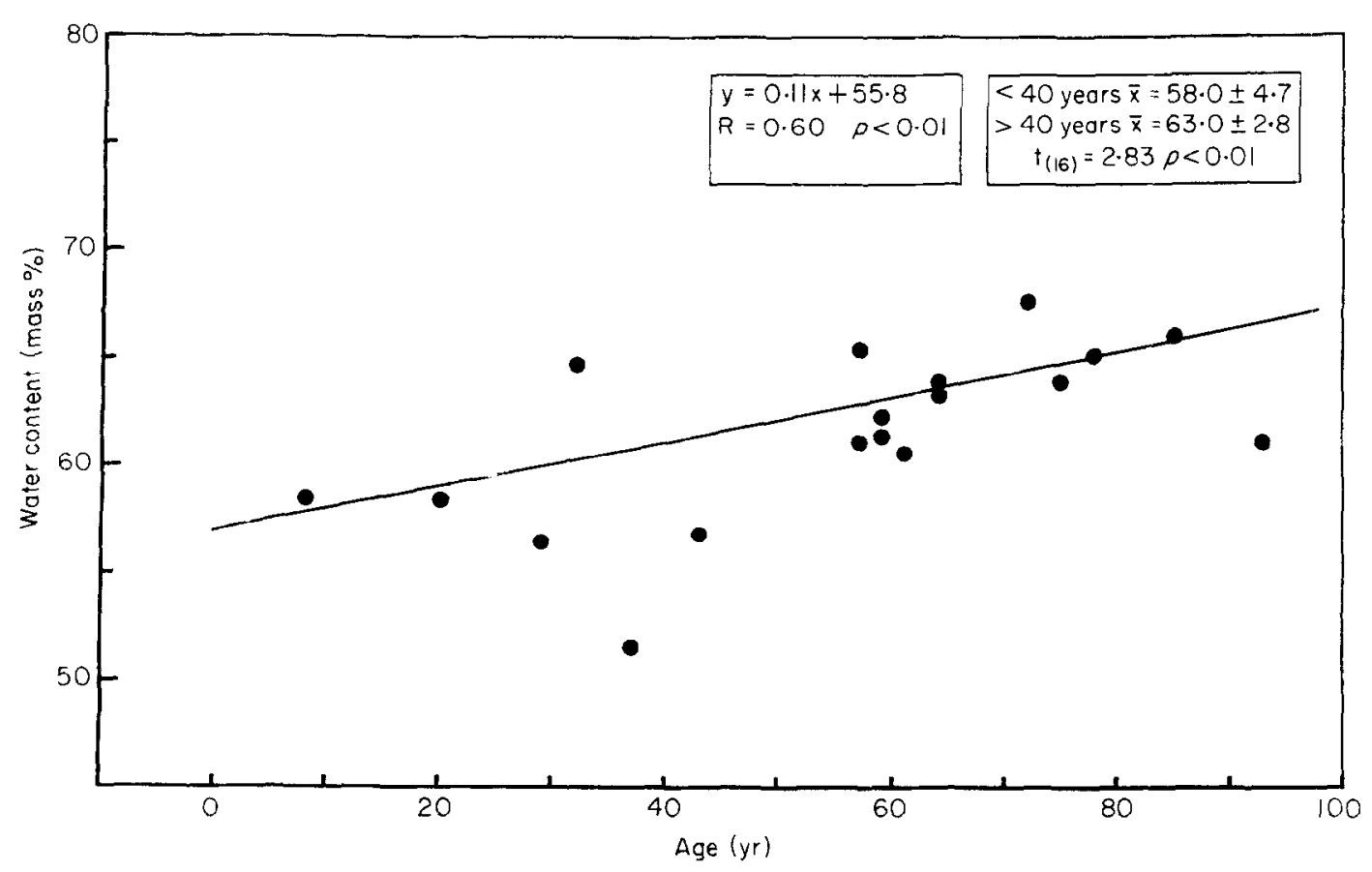

FIG. 4. Mean nuclear water content of the human lens as a function of age. 
human lens with small opacities. The straight line indicates the plane of slice-cutting. In Fig. 2(B) a micrograph of the lens slice is shown. On dissection this type of opacity proved to extend uninterrupted in the anterior and posterior direction, as described by Willekens. Kappelhof and Vrensen (1987). In order to compare the water content in the turbid spot to the according region on the opposite side we also measured the water content along the equatorial axis in this region using the same procedure as described above.

For statistical testing the Student's $t$-test, the Mann-Whitney test and the least-squares linear regression method were used.

\section{Results}

Figure 3(A) exemplifies the water content data along the visual axis of a human lens, 8 years of age. There was a steep drop in water content in the anterior and posterior pole which is in agreement with the results of a previous study (Huizinga et al., 1989). The water content in the nucleus was constant. The drop in water content in the equatorial pole proved to

\section{TABLE I}

Water content in the outermost anterior and posterior poles of the visual axis

\begin{tabular}{ll}
\hline Anterior & $85 \cdot 3 \% \pm 9 \cdot 41 \%(18)^{*}$ \\
Posterior & $80 \cdot 9 \% \pm 8.32 \%(18)$ \\
\hline
\end{tabular}

* Mean, standard deviation and number of lenses analysed. Statistical significance: (1) Student's $t$-test: $t(17)=1 \cdot 61,0 \cdot 10>P$ $>0.05$; (2) Mann-Whitney test: ties 282-384, 0.05 >P>0.025. be more gradual. This is illustrated in the equatorial axis profile of the same 8-year-old human lens [Fig. $3(\mathrm{~B})]$.

The results of the mean nuclear water values are given in Fig. 4. The mean nuclear water content of human lenses was linearly correlated with age. This correlation was highly significant with a correlation coefficient, $R$, of $0.60(P<0.01)$. Based on the results of physiological (Duncan et al., 1989) and ultrastructural studies (Vrensen and Willekens. 1990) the lenses were arbitrarily divided into two groups: below and above 40 years of age. The water content in the nucleus proved to be significantly higher in lenses of donors over the age of 40 years $(P<0 \cdot 0)$. Student's $t$-test). with a mean value and standard deviation of $58 \cdot 0 \pm 4.7$ (mass \%) for the first and a mean value and standard deviation of $63 \cdot 0 \pm 2 \cdot 8$ for the latter group.

From the data on water content in the anterior and posterior pole (Table 1) there seemed to exist a slight difference between the outermost anterior and the outermost posterior value with a lower value in the latter. A paired Student's $t$-test indicated a significance level of $0.05<P<0.10$ and using the MannWhitney test a $P$ level of $0.025<P<0.05$ was found.

Age-related changes in the water-content gradient in the anterior and posterior pole and along the equatorial axis of the human lens, expressed as the width of the surface layer, are given in Fig. 5. By leastsquares regression the age dependency of the surface layer width was tested. In the equatorial pole the surface layer width significantly decreased with age $(R=0.88 ; P<0.01)$. In the anterior and the posterior pole no significant age-related changes in the surface layer width were observed.

The results of the water content measurements

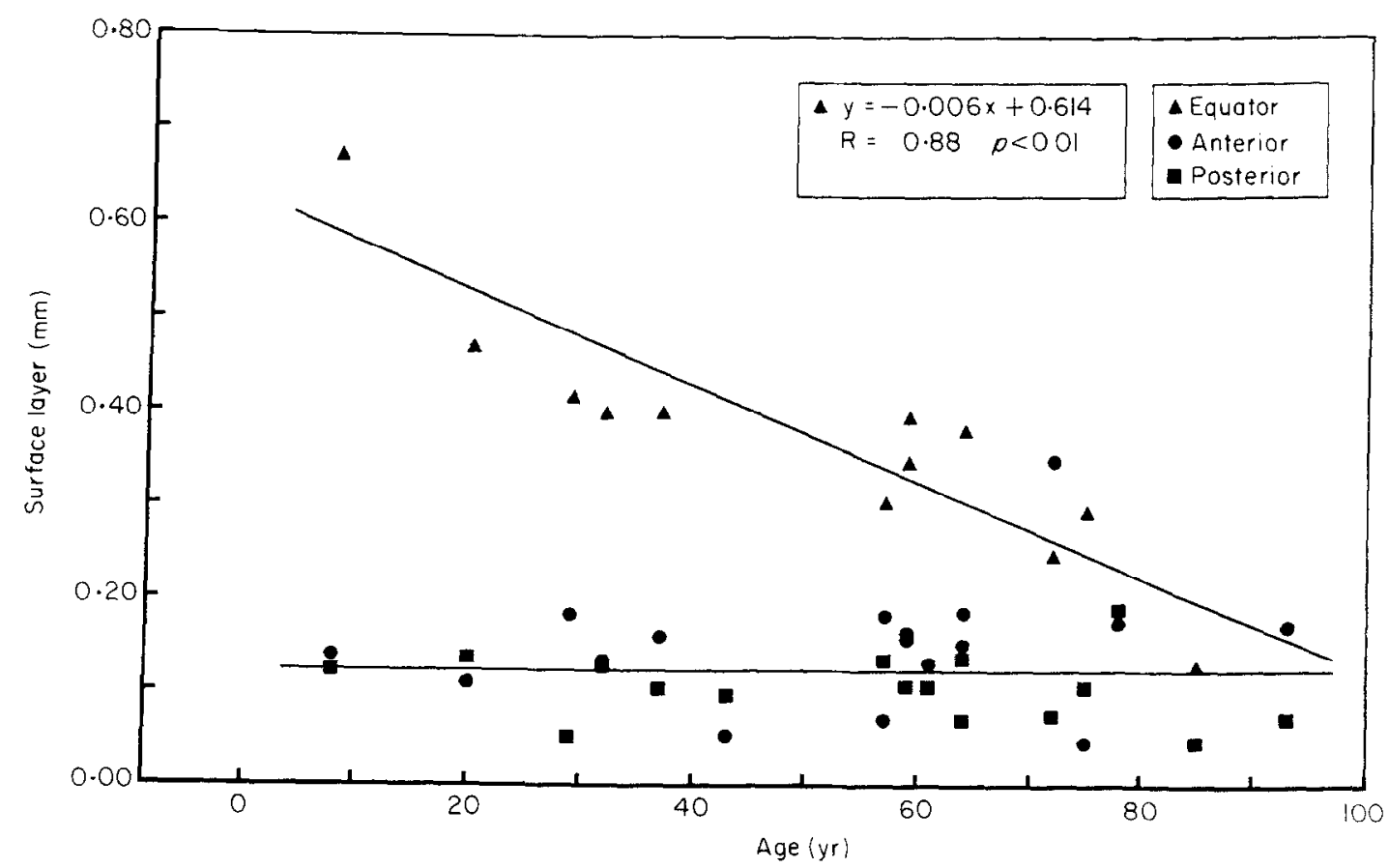

FIG. 5. Surface layer width in the equatorial 


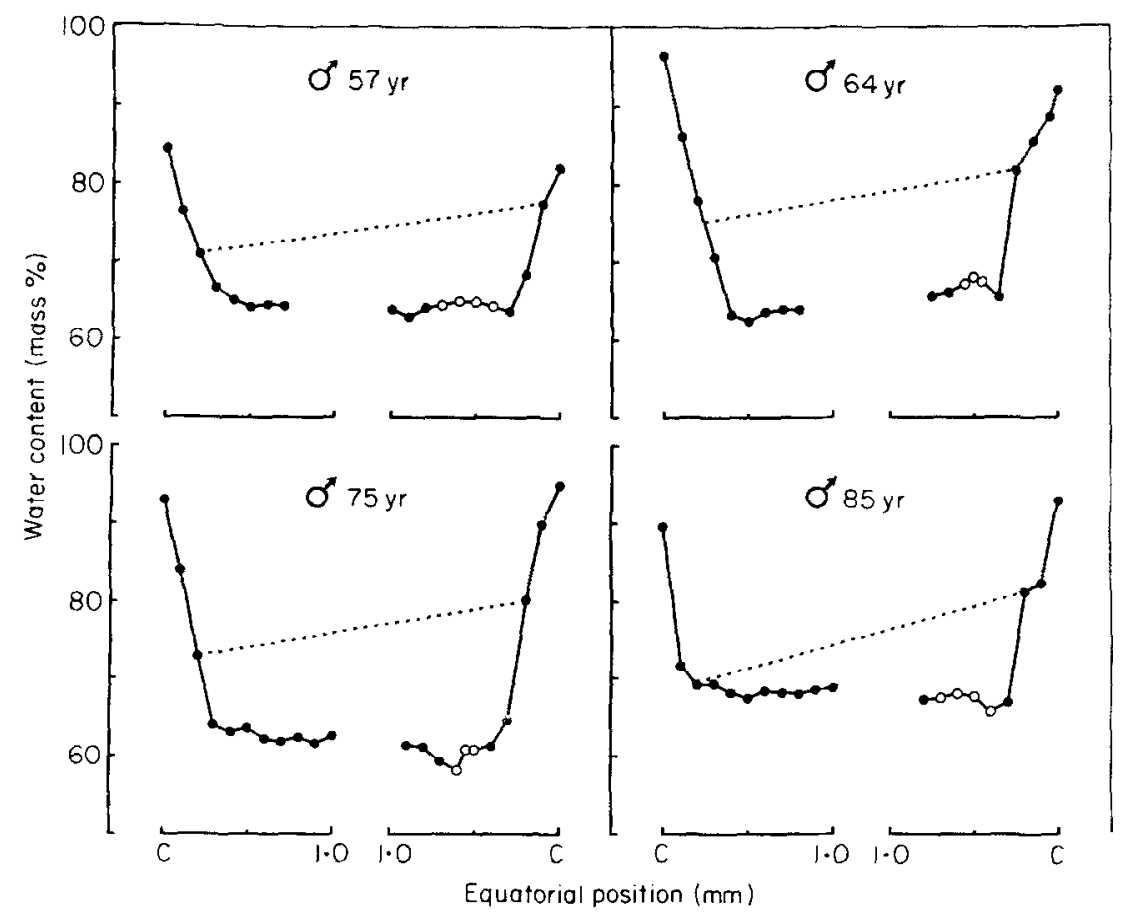

FIG. 6. Absolute water content along the equatorial axis in four human lenses with small opaque spots in their equatorial region. The open symbols are representing the data points obtained inside the opaque spots. Note the normal-for-site water content in the spots. The dotted lines are connecting a position on the equatorial axis to a similar position on the opposite side. This illustrates the zone with a high-for-site water content at the capsular side of the opaque spots.

along the equatorial axis of four out of the seven human lenses with small opaque spots in their equatorial region are exemplified in Fig. 6. In all lenses studied the opaque spots had a normal-for-site water content. But in six out of the seven lens-slices a zone with a high-for-site water content at the capsular side of the opaque spot was found. This is illustrated by the dotted lines in the individual measurements which are connecting a position on the equatorial axis to the similar position on the opposite side.

\section{Discussion}

Vacuum dehydration and freeze drying have been used to investigate the water/protein content of clear and cataractous human lenses (Van Heyningen, 1972: Fisher and Pettet, 1973; Bettelheim et al., 1986a.b; Deussen and Pau, 1989). As outlined previously (Huizinga et al., 1989) and confirmed in the present paper nuclear protein values (35-39\%) thus obtained for clear human lenses are in good agreement with values obtained with Raman microspectroscopy (35\%) emphasizing the quantitative reliability of the Raman procedure used in this and the previous studies (Huizinga et al., 1989; Bot et al.. 1989).

In contrast to bovine, rabbit and rat lenses in which the protein content gradually increases up to the deep nucleus (Huizinga et al., 1989; Philipson, 1969; Van Heyningen. 1972) the main body of the clear human lens has a constant and relatively low protein content (Huizinga et al., 1989; Fagerholm, Philipson and
Lindström, 1981 and this paper). As shown in this paper this constant nuclear value is reached within $0.05-0.2 \mathrm{~mm}$ from the anterior and posterior surfaces. Steep gradients such as this can only be appreciated using a strict local approach with a small measuring volume. The dry-weight microsectioning technique of Bours, Födisch and Hockwin (1987) also indicates a steep drop in water content from outer cortex to nucleus but the tissue volumes used are still too large to appreciate fully the steepness of the gradient. Fagerholm, Philipson and Lindström (1981) used a carefully calibrated quantitative microradiographic method on freeze-dried sections of lenses. The subcapsular $(20 \%)$ and constant nuclear $(37 \%)$ protein values correspond extremely well with the Raman values presented in this paper $(15-20 \%$ and $38 \%$ respectively) emphasizing once more the quantitative reliability of the Raman procedure used. However, regarding the steepness of the anterior and posterior gradients considerable differences between both methods exist. Values of 0.4 and $0.6 \mathrm{~mm}$ were observed in the microradiographic approach for anterior and posterior gradients respectively whereas in the present Raman study values ranging from 0.05 to $0.2 \mathrm{~mm}$ were observed with no significant difference between anterior and posterior poles.

As indicated by Philipson (1969) a major error introduced by freeze drying is shrinkage of the tissue. Philipson and Lindström (1969) estimated this shrinkage to be $3-4 \%$ in all dimensions causing an increased mass per unit volume of about $10 \%$. The gradient values of 0.4 and $0.6 \mathrm{~mm}$ were calculated by correct- 
ing the basic measurements for this, assuming equal shrinkage throughout the lens. However, since shrinkage is dependent on protein concentration this assumption is not fully valid and it is therefore not inconceivable that the gradient values are overestimated. It was shown (Bot et al., 1989; Huizinga et al., 1989) that fixation with a nearly isoosmotic ( $310 \mathrm{mosmol}$ ) cacodylate-buffered paraformaldehyde solution followed by slicing had only a very minor effect on the water/protein gradient. Therefore, it seems likely that the present gradient values are very close to reality.

The steep gradients, expressed as surface layer width, proved to be independent of age in the visual axis (Fig. 5). However, along the equatorial axis the surface layer width significantly decreased with age (Fig. 5). The equatorial bow region represents the central parts of the lens fibres still containing cell nuclei and numerous cell organelles and these parts are metabolically active. The anterior and posterior parts of these fibres are largely devoid of these organelles and already contain large amounts of crystallins. This explains the difference in surface layer width between the anterior and posterior surfaces and the equatorial surface in young lenses. As outlined by Kuwabara (1975) mitotic activity is reduced upon aging leading to a decrease of differentiating and maturing bow cells. The age-related decrease in equatorial surface layer width is in agreement with this observation.

Systematic studies on age-related changes in the nuclear water/protein content of human lenses are scarce. The microradiographic study of Fagerholm. Philipson and Lindström (1981) did not reveal significant changes in nuclear protein content with age. However, the protein-dependent increase in mass per unit volume due to freeze drying, as oullined above, could have masked small differences. The study of Nordmann (1973) did not show an age-related regression of nuclear dry weight after drying at $110^{\circ} \mathrm{C}$ or lyophilization, in human lenses over the age of 40 years. The author states, however, that in some instances nuclear dry weight is about equal to the cortical dry weight indicating an excessive hydration of the nucleus. Re-evaluation of the data given by Van Heyningen (1972), using linear regression analysis, showed a slight but significant $(R=0.60, n=15)$ tendency of decreased dry weight in the lens nucleus in old age, thus supporting the observation of the present Raman study.

Increase in nuclear water content affects the refractive power of the lens especially when the increase concerns such a large volume as in the human lens. Refractive index gradients along the equatorial and visual axis measured by tracing laser beam paths in bovine and rat lenses (Campbell, 1984; Pierscionek, 1989: Pierscionek and Augusteyn, 1990) markedly resemble the protein distribution curves of rabbits (Bot et al.. 1989; Huizinga et al., 1989) supporting the view that protein distribution and refractive index are closely correlated. IJpon aging the lens thickness (posterior-anterior dimension) increases whereas the equatorial diameter remains constant over the age of 30 years (cf. Willekens et al., 1987). In addition it has been found that the curvatures of the anterior and posterior poles become sharper and that the entire lens nucleus is shifted towards the cornea (Brown, 1973, 1974; Koretz, Handelman and Brown, 1984; Koretz and Handelman, 1986; Koretz, et al., 1989). These changes in lens morphology and position theoretically result in a loss of far vision with age. In fact the opposite is the case: aging is accompanied by a reduction in near vision. This discrepancy is known as the Lens Paradox. Koretz and Handelman (1988) discussing this Lens Paradox made two suggestions to explain this: (i) a reduction of the refractive index, and/or (ii) a shallowing of the refractive index gradient with age. The present results, and the supposed correlation between protein content and refractive index, are in strong support of the first suggestion. The absence of an age-related change in surface layer width does not favour the second suggestion. It will be evident that further studies are needed to verify to what extent this increased nuclear water content can account for the Lens Paradox.

The age-related increase in nuclear water content coincides with other age-related changes in the physiological and biochemical properties of the human lens. Numerous post-translational changes in lens crystallins have been described as e.g. aggregation, insolubilization, disulphide formation and deamidation (cf. Hoenders and Bloemendal, 1981). These posttranslational changes in crystallins, most evident in the nucleus, could induce changes in the colloid osmotic pressure. Duncan et al. (1989) recently described an increase in cation permeability in aging human lenses leading to a rise in $\mathrm{Na}^{\prime}$ and $\mathrm{Ca}^{2+}$. These ionic changes could disturb the water equilibrium in the aged lens. Finally, upon aging the pumping capacity of the epithelium and superficial cortical fibres will be reduced. These three factors will all have an effect on the water equilibrium in the lens and could be responsible for the increased water content in the nucleus of the aged human lens.

The observation that opaque spots have a normalfor-site water content is unprecedented. Recent scanning electron microscopic and frecz fracturc investigations (Vrensen and Willekens, 1990; Vrensen, et al., 1990) have shown that these early cortical opacitics are segregated from non-affected parts of the lens. They are filled with membrane-bound vesicles and their membranes have a deviating globular aspect. In freeze fracture the membranes proved to have no gap junctions and intramembrane particles and to be rich in square arrays known to be involved in uncoupling of the cells from their environment. Whether these membrane changes lead to abnormal permeability 
properties protecting these spots for uncontrolled influx of water or whether this membrane alteration leads to uncontrolled water influx counteracted by the osmotic pressure of the surrounding normal fibres needs to be further investigated. This also holds for the high-for-site water content at the capsular site of the early opacities. It may be that the high phosphorus and low sulphur content of these opaque spots as found by Harding et al. (1982) is related to this problem, but certainly needs further study.

\section{Acknowledgements}

The authors are indebted to Dr E. Pels of the Cornea Bank, Amsterdam for collecting the lenses and to Mr B. Willekens for technical assistance. We thank Mr N. Bakker for adapting the figures. The authors gratefully acknowledge the constructive contribution of Dr Jane Koretz with respect to the points raised in the discussion. This study was carried out within the framework of the European Economic Community Concerted Action on Cellular Aging and Diseases 'Eurage'.

\section{References}

Bettelheim, F. A., Ali, S., White, O. and Chylack, L. T. (1986a). Freezable and non-freezable water content of cataractous human lenses. Invest. Ophthalmol. Vis. Sci. 27. $122-5$.

Bettelheim, F. A.. Castoro, J. A.. White, O. and Chylack, L. T. (1986b). Topographic correspondence between total and non-freezable water content and the appearance of cataract in human lenses. Curr. Eye Res. 5, 925-32.

Bot, A. C. C., Huizinga, A., de Mul, F.F.M., Vrensen, G. F. J. M. and Greve, J. (1989). Raman microspectroscopy of fixed rabbit and human lenses and lens slices: new potentialities. Exp. Eye Res, 49, 161-9.

Bours, J., Födisch, H. J. and Hockwin. O. (1987). Age-related changes in water and crystallin content of the fetal and adult human lens, demonstrated by a microsectioning technique. Ophthalmic Res. 19, 235-9.

Brown. N. (1973). The change in shape and internal form of the lens of the eye on accommodation. Exp. Eye Res. 15. 441-59.

Brown, N. (1974). The change in lens curvature with age. Exp. Eye Res. 19, 175-83.

Campbell, M. C. W. (1984). Measurement of refractive index in an intact crystalline lens. Vision Res, 24, 409-15.

Deussen, A. and Pau, H. (1989). Regional water content of clear and cataractous human lenses. Ophthalmic Res. 21. 374-80.

Duncan, G., Hightower, K. R., Gandolfi, S. A., Tomlinson, J. and Maraini, G. (1989). Human lens membrane cation permeability increase with age. Invest. Ophthalmol. Vis. Sci. 30, 1855-9.

Fagerholm, P. P., Philipson, B. T. and Lindström, B. (1981). Normal human lens - the distribution of protein. Exp. Eye Res. 33, 615-20.

Fisher, R. F. and Pettet, B. E. (1973). Presbyopia and the water content of the human crystalline lens. J. Physiol. 234. 443-7.
Gijsbers, G., Vrensen, G., Willekens, B., Maatman, D., de Mul. F. and Greve. J. (1986). Raman microspectroscopic. investigations of human eye lenses. In Laser Scattering Spectroscopy of Biological Objects. (Eds. Stepanec, I.. Anzenbacher. P. and Sedlacek, B.). Pp. 583-594. Elsevier: Amsterdam.

Harding, C. V., Chylack, L. T., Susan, S. R., Lo. W.-K. and Bobrowski, W. F. (1982). Elemental and ultrastructural analysis of specific human lens opacities. Invest Ophthalmol. Vis. Sci. 23, 1-13.

Hoenders, H. J. and Bloemendal, H. (1981). Aging of lens proteins. In Molecular and Cellular Biology of the Eye Lens. (Ed. Bloemendal, H.). Pp. 279-326. John Wiley \& Sons: New York.

Huizinga, A., Bot, A.C. C., de Mul. F. F. M., Vrensen, G. F. J. M. and Greve, J. (1989). Local variation in absolute water content of human and rabbit eye lenses measured by Raman microspectroscopy. Exp. Eye Res. $48,487-96$.

Koretz, J.F. and Handelman, G. H. (1986). The 'lens paradox' and image formation in accommodating human eyes. In The Lens: Transparency and Cataract. (Ed. G. Duncan) Topics in Aging Research in Europe 6. 5764 .

Koretz, J. F. and Handelman, G. H. (1988). How the human eye focuses. Scientific American 256 (7), 64-71.

Koretz, J. F.. Handelman, G. H. and Brown. N. P. (1984). Analysis of human crystalline lens curvature as a function of accommodative state and age. Vision Res. $24,1141-51$

Koretz. J. F.. Kaufman. P. L., Neider. M. W. and Goeckner. P. A. (1989). Accommodation and presbyopia in the human eye-aging of the anterior segment. Vision Res. 29, $1685-92$

Kuwabara, T. (1975). The maturation of the lens cell: a morphologic study. Exp. Eye Res. 20, 427-43.

Lahm, D., Lee, L. K. and Bettelheim, F. A. (1985). Age dependence of freezable and nonfreezable water content of normal human lenses. Invest. Ophthalmol. Vis. Sci. 28. $1162-5$

Nordmann. J. (1973). Le noyau du cristallin. I-Ia teneur en eau. Arch. d'Ophthalmol. 33, 81-6.

Philipson, B. (1969). Distribution of protein within the normal rat lens. Invest. Ophthalmol. 8, 258-70.

Philipson, B. and Lindström, B. (1969). Specimen preparation for quantitative microradiography. Histochemie 17. 201-6.

Pierscionek, B. K. (1989). Growth and aging effects on the refractive index in the equatorial plane of the bovine lens. Vision Res. 29, 1759-66.

Pierscionek, B. K. and Augusteyn, R. C. (1990). Age-related changes in the refractive index and protein distributions in the lens. Proc. Int. Soc. Eye Res. 6, 36.

Van Heyningen. R. (1972). The human lens. III. Some observations on the postmortem lens. Exp. Eye Res. 13, 155-60.

Vrensen, G., Van Marle. J., Willekens, B. and Van Veen, H. (1990). Square arrays in early cortical lens opacities. Invest. Ophthalmol. Vis. Sci, 31, 279-84.

Vrensen, G. and Willekens, B. (1990). Biomicroscopy and scanning electron microscopy of early opacities in the aging human lens. Invest. Ophthalmol. Vis. Sci. 31 , $1582-91$.

Willekens, B., Kappelhof, J. and Vrensen, G. (1987). Morphology of the aging human lens. I. Biomicroscopy and biometrics. Lens Res. 4, 207-30. 